\title{
我国激光技术医疗应用和产业发展战略研究
}

\author{
邱海霞 $^{1}$, 李步洪 ${ }^{2}$, 马辉 ${ }^{3}$, 魏勋斌 ${ }^{4}$, 杨思华 ${ }^{5}$, 李阳 ${ }^{6}$, 谭一舟 ${ }^{1}$, 陈德福 ${ }^{7}$, \\ 赵洪友 ${ }^{7}$, 曾晶 ${ }^{1}$, 顾瑛 ${ }^{1}$
}

(1. 中国人民解放军总医院第一医学中心，北京 $100853 ； 2$. 医学光电科学与技术教育部重点实验室暨福建省光子技术重点 实验室 (福建师范大学), 福州 350117; 3. 清华大学深圳国际研究生院, 广东深圳 518055; 4. 北京大学跨学部生物

医学工程系, 北京 $100871 ;$ 5. 华南师范大学生物光子学研究院暨激光生命科学教育部重点实验室, 广州 510631;

6. 北京镭科光电科技有限公司, 北京 100192； 7. 北京理工大学医工融合研究院，北京 100081）

摘要: 激光诊疗技术在生命健康领域得到了越来越多的成功应用, 成为现代医学精准诊疗的重要组成部分。本文针对激光技 术医疗应用主题, 重点从实际应用和产业链的视角出发, 梳理我国激光技术在临床治疗、诊断和产业化方面的发展现状、前 景趋势和存在问题。研究发现, 尽管我国在激光治疗技术、医用激光诊疗设备及其产业发展方面均取得了长足进步, 但整体 而言, 激光医疗技术的临床应用仍处于跟踪起步和同步发展阶段, 原创性应用研究有所缺乏, 医用激光设备的研发和产业化 处于中低端, 用于精准诊疗的超快激光器等关键技术和高端设备仍未打破国外垄断。研究建议, 设立我国激光医学二级学科, 加强国家级激光医疗科技创新平台建设, 针对性开展激光医疗器械法规建设, 出台相关政策扶持激光医疗器械产业发展, 组 织攻关医用激光诊疗设备关键核心技术。

关键词：激光技术; 医疗; 激光诊断; 激光治疗; 激光产业

中图分类号: R318.51 文献标识码: A

\section{Medical Application and Industrial Development Strategy of Laser Technology in China}

\author{
Qiu Haixia ${ }^{1}$, Li Buhong ${ }^{2}$, Ma Hui ${ }^{3}$, Wei Xunbin ${ }^{4}$, Yang Sihua ${ }^{5}$, Li Yang ${ }^{6}$, Tan Yizhou ${ }^{1}$, \\ Chen Defu ${ }^{7}$, Zhao Hongyou ${ }^{7}$, Zeng Jing ${ }^{1}$, Gu Ying ${ }^{1}$
}

(1. First Medical Center of Chinese PLA General Hospital, Beijing 100853, China; 2. MOE Key Laboratory of OptoElectronic Science and Technology for Medicine, Fujian Provincial Key Laboratory for Photonics Technology, Fujian Normal University;

Fuzhou 350117, China; 3. Tsinghua Shenzhen International Graduate School, Shenzhen 518055, Guangdong, China;

4. Biomedical Engineering Department, Peking University, Beijing 100871, China; 5. MOE Key Laboratory of Laser Life Science \& Institute of Laser Life Science, College of Biophotonics, South China Normal University, Guangzhou 510631, China; 6. Beijing Laserconn Technology Co., Ltd., Beijing 100192, China;

7. Institude of Engineering Medicine, Beijing Institute of Technology, Beijing 100081, China)

\begin{abstract}
Laser diagnosis and treatment technology has been successfully applied in the field of healthcare and become an important part of precision diagnosis and treatment in modern medicine. This article focuses on the medical application of laser technology and summarizes the development status, trends, and problems of China's laser technology in clinical treatment, diagnosis, and industrialization, from the perspectives of practical application and industrial chain. The study revealed that China's laser medicine
\end{abstract}

收稿日期 : 2020-03-25; 修回日期 : 2020-05-14

通讯作者: 顾瑛, 中国人民解放军总医院第一医学中心主任医师、教授, 中国科学院院士, 研究方向为激光/光疗在临床医学的精准诊疗应 用; E-mail: guyinglaser301@163com

资助项目：中国工程院咨询项目 “我国激光技术与应用 2035 发展战略研究” (2018-XZ-27)

本刊网址： www.engineering.org.cn/ch/journal/sscae 
has made great progress in terms of laser treatment technology, medical laser diagnosis and treatment equipment, and the industry; however, on the whole, the clinical application of laser medical technology is still catching up with the global trend. The applied research of the technology still lacks original creativity, and the research and industrialization of medical laser equipment are at the mid-low end. The key technologies and high-end equipment such as ultrafast lasers for precision diagnosis and treatment are still under foreign monopoly. The study proposes that China should establish sub-disciplines under laser medicine; strengthen the construction of national science and technology innovation platforms for laser medicine; develop regulations on laser medical equipment in a targeted manner; launch relevant policies to support the development of the laser medical equipment industry; and strive to make breakthroughs in key technology research for medical laser diagnostic equipment.

Keywords: laser technology; medicine; laser diagnosis; laser therapy; laser industry

\section{一、前言}

自 1960 年世界上第一台红宝石激光器面世以 来, 这一新型光源和随之产生的新型激光技术开始 应用于医学领域。经过 60 年的发展, 激光医学已初 步发展成为一门体系较为完整且相对独立的新型交 叉学科, 在医学科学中起着越来越重要的作用。目 前, 激光医学在临床应用上已经形成了强激光治疗、 光动力治疗 (PDT)、弱激光治疗 (LLLT) 三大 激光治疗技术 [1], 同步发展了包括光学相干层析 成像 (OCT)、光声成像、多光子显微成像、拉曼 成像在内的众多兼具高灵敏度和高分辨率的激光诊 断技术 [2]。此外, 随着化学和生物学等相关学科 的发展, 各种生物探针和靶向标记技术得到快速发 展, 激光光学、材料学、纳米技术和生物技术的相 互融合正在不断地为医学诊断和治疗带来新的发展 空间。

随着现代医学模式的转变, 激光医学的应用领 域正从疾病诊疗向疾病预防前移, 以精准性、微 创性、无创性为技术突破方向来引领医学诊疗模 式的转变。激光诊疗技术的发展与应用促进了医 用激光设备的产业化, 国际上已经形成较为完善 的医用激光设备产业。与发达国家相比, 我国医 用激光在核心技术、产业规模、推广应用等方面 尚存在一定的差距, 高端激光医疗器械市场长期 被进口产品占据，相应技术服务能力也未能全面 满足国民医疗的需求。

本文系统调研我国激光技术在临床治疗、诊断 和激光医疗产业中的发展现状、前景趋势、存在问 题, 提出加快我国激光医疗技术与应用产业发展的 措施建议, 以期为国产技术装备及其医疗应用提供 发展参考。

\section{二、激光技术医疗应用现状分析}

\section{（一）激光诊断技术}

激光诊断技术利用激光的高单色性、光强度高、 准直性、偏振性等光学基本属性以及光与物质的各 种相互作用（散射、吸收等）来测量生物组织的微 观结构、生理作用、生化分子浓度分布等关键指标, 获取生物组织的结构和功能信息, 剖析疾病的发生 发展过程。凭借无损成像、高分辨率和丰富的对比 机制等优势, 激光诊断技术成为现代医学精准诊疗 的重要组成部分。

随着激光技术的不断进步和临床精准诊疗需求 的持续牵引, 激光成像方面的新技术、新机理、新 概念不断涌现, 衍生出了非接触无标记成像、实时 在体成像等诊断新技术，逐步走向临床应用。典型 的有光学相干断层成像 (OCT) [3]、光声成像 [4]、 激光散斑成像 [5]、多光子显微成像 [6]、共聚焦成 像 [7]、拉曼成像 [8] 等。OCT 作为代表性的光学诊 断技术, 以无损伤的近红外光为光源, 实时在体获 得类似于组织病理的视网膜断层图像, 广泛应用于 眼科多种疾病的诊断。近年来, OCT 也被拓展应用 于眼科之外的科室, 如皮肤科和口腔科。随着 OCT 设备的小型化及其与导管、内窥镜的融合, 未来在 心血管疾病、胃肠道疾病、肿瘤早期诊断等方面的 应用有望进一步加强。

上述激光成像方法尽管在生物医学研究方向发 挥了重要作用，但不可避免地存在一定的应用局限 性：单一模态成像手段通常只能获取部分信息，而 不同模态光学成像方法获取的光学信息存在着差 异。针对特定疾病, 综合不同模态光学成像方法的 分析结果, 形成多模态、多维度的光学检测和监测 平台, 这是未来激光诊断技术的重点发展方向。激 
光诊断技术的应用范围将继续拓宽，从定点照护和 实验室检测, 到篎查、诊断成像和治疗监测, 再到 手术过程中的实时在体成像及肿瘤边界识别等。未 来, 借助基因工程方法, 植入光电子和细胞内器件 (如微米和纳米激光器) 来增加患者对光敏功能的 整合, 从而进一步拓展激光诊断技术应用。

\section{（二）激光治疗技术}

\section{1. 强激光治疗}

强激光治疗是利用激光的光热效应, 对生物 组织进行凝固、汽化或切割来达到消除病变的目 的 [9]。自 20 世纪 60 年代红宝石激光成功用于视 网膜脱落的光凝治疗以来 [10], 强激光治疗因其出 血少、操作定位精确、非接触、无菌、对周围组织 损伤小等优点, 在临床应用中迅速得到扩大, 成为 激光医疗发展最快、最为成熟的分支。强激光治疗 已经广泛用于眼科、皮肤科、泌尿外科、消化科、 口腔科、耳鼻喉科等 [1], 作为 “光刀” 使用改变 了传统手术方式, 对某些难治性疾病的治疗实现了 革命性突破。尤其在眼科领域, 强激光治疗作为现 代眼科的关键技术, 被视为多种眼部疾病的首选治 疗方案。

以超快激光为代表的前沿激光技术 (如皮秒激 光、飞秒激光) 具有更高选择性、更精准切割等特 点, 逐渐在医疗应用和生命科学中显示出了应用潜 力 [11]。飞秒激光在透明生物组织中可以无衰减地 传输到聚焦点, 对周围组织热损伤小且切割精度高。 与传统治疗手术和其他激光手术相比, 飞秒激光手 术具有更高的准确性、安全性和稳定性, 被视为相 对完美的临床眼科治疗方法。随着眼科、精准治 疗等医学需求带动以及手术机器人技术的迅猛发 展, 医用超快激光器等新型强激光应用有望形成 较大的产业规模, 进而为某些难治性疾病的治疗 提供新手段。

\section{2. 光动力治疗}

PDT 是继手术、化疗和放疗之后形成的一种治 疗肿瘤的新型微创疗法。在 PDT 过程中, 光敏剂 在特定波长激光的激活下出现了一系列光物理化学 反应, 产生具有生物毒性的活性氧物质来杀伤靶组 织, 进而实施靶向治疗 [12]。与传统治疗手段相比, PDT 具有选择性高、微创、可重复使用、无耐药性、 能最大限度保留组织和器官完整性等优点 [13]。随
着对 PDT 作用机制研究的不断深入, 目前 PDT 的 适应证已从起初的肿瘤治疗逐渐拓展到肿瘤靶向 PDT、血管靶向 PDT、微生物靶向 PDT 三大治疗 领域, 在恶性肿瘤及癌前病变、难治性微血管病变 (如老年性眼底黄斑变性、鲜红斑痣、胃窦血管扩 张等)、难治/耐药性微生物感染等方面具有良好的 应用前景。

PDT 疾病治疗谱和疗效与其所采用光的波长、 强度、照光方式等光参量密切相关, 因而光源是 PDT 的关键构成。在早期应用阶段, 通常采用白炽 灯、高压弧形灯等非相干光作为辐射光源, 这类光 源在光谱结构、功率密度、传输系统、精确控制等 方面存在着不足。鉴于激光技术的独特优势, 激光 已经成为 PDT 首选光源, 显著提升了 PDT 临床应 用的效果。

PDT 作用机制独特而复杂, 治疗谱范围广泛, 且具体治疗靶点特征各异。针对不同靶点疾病开展 系统深入的研究来取得新的治疗突破, 这是当前激 光治疗最为活跃的研究方向, 相应热点主要有: 高 靶向性、高选择性的功能型光敏剂研发, PDT 治疗 深度提升 (如双光子 PDT, 上转换纳米材料 PDT 等) $[14,15]$, PDT 新型光源研发与应用, PDT 新 适应症机制与量效研究, PDT 治疗中光剂量精准 调控等。

\section{3. 弱激光治疗}

LLLT 又称低强度激光治疗或光生物调节治疗, 是指激光作用于生物组织时不造成不可逆的损伤, 但刺激机体产生一系列的生理生化反应, 对组织或 机体起到调节、增强或抑制作用来达到治疗疾病的 目的 $[16,17]$ 。LLLT 的最大特点是患者无创无痛, 其功率密度通常为毫瓦量级。自 20 世纪 70 年代 起, LLLT 临床应用在东欧、苏联和我国较为广泛。 随着半导体激光器的发展, 包括红光和近红外光在 内的多种波长激光被用作 LLLT 治疗光源, 用于内 科、外科、妇科、儿科、眼科、耳科、口腔科等 临床科室的 300 多种疾病治疗, 对促进伤口愈合、 疼痛缓解、炎症消退、组织再生、肌肉疲劳缓解 等具有良好功效。目前, 临床用激光器主要为 $\mathrm{He}$ $\mathrm{Ne} /$ 半导体激光器, 波长为红光波段 (630 690 nm) 和近红外波段 (760 940 nm), 主要采用连续输出 模式。

激光波长、激光剂量、连续或脉冲激光输出模 
式会产生不一样的生物调控过程。随着 LLLT 作用 机制的深入研究、新光源的涌现与应用, LLLT 应 用领域也在不断拓宽，除临床成熟应用（如感染、 炎症、疼痛）之外，紫外及近红外脉冲激光在一些 重大慢性病及增龄性疾病中的治疗和预防方面展现 了良好前景。近年来, LLLT 在神经退行性疾病的 预防和治疗方面的探索取得一定进展。在目前没有 任何安全有效的方法来治疗神经退行性疾病的背景 下, LLLT 开辟了一个具有前景的新方向, 有望驱 动弱激光临床应用及相关激光技术的进一步发展。

随着医学模式的转变、LLLT 疾病谱从常见病 向重大慢性增龄性疾病的拓展，相关治疗领域正在 从疾病治疗向疾病预防延伸, 应用主战场也出现 了由医疗机构向社区和家庭的转变。这种形势对 LLLT 治疗设备的便携性、小型/微型化、可穿戴 化提出了新的更高要求。可以预期, 基于激光技术 的可穿戴设备将在疾病治疗方面发挥更为普遍和重 要的作用。

\section{（三）激光监测技术}

基于发光二极管（LED）光源的医学监测技术, 因其能够监测血糖、血氧等重要生理指标而逐步兴 起。相比于 LED 光源, 激光光源具有更好的光学 特性, 能够提供全新的无损、精准监测手段: 实现 微创或无创监测, 兼具高灵敏度、高选择性和长期 稳定性, 显著提高医学监测结果的精准性。随着医 学治疗模式的转变, “医院治疗 + 家庭健康监护” 模式将是发展潮流, 而精准激光监测技术正在成为 医学监测设备的重要发展方向。

在高灵敏激光健康监测方面, 基于呼吸气体、 尿液和血液等的激光监测技术是未来发展重点。

在微型化激光健康监测方面, (1)发展便携式激 光监测技术, 将激光监测系统进行小型化和集成化 处理，如便携式血糖血压检测仪; (2)开发可穿戴激 光监测技术, 设计适合人体穿戴的激光监测设备, 如可穿戴智能激光监测手表; (3)发展内窥式激光监 测技术, 将开展分子、细胞和组织层次检测的激光 技术与目前成熟的内窥镜技术相结合, 用于体内组 织的实时监测。

在智能化激光健康监测方面, (1)发展基于大数 据的激光监测功能, 人工智能技术将成为自动化处
理与分析生物医学大数据的有力工具; (2)针对动态 医学检测需求, 发展体积小、重量轻、电压和功耗 低的激光光源, 提高诊断激光器的物理指标及设备 续航性来满足用户体验要求; (3)未来的激光诊疗方 法朝着植入式方向发展, 探索微纳激光等新型激光 器的生物相容性、可降解性。

\section{三、我国激光医疗产业发展态势}

根据 Allied Market Research 市场调研报告 [18], 2016 年世界激光医疗市场达到 51.16 亿美元, 预 计 2023 年将增加到 125.86 亿美元, 年均增长约为 $13.6 \%$; 美国、欧洲、以色列和日本位居世界领先 地位。关于激光医疗器械的全球市场份额, 北美洲 约占 $35 \%$, 欧洲及中东约占 $25 \%$, 亚太区域约占 $20 \%$, 我国约占 $15 \%$ 。国际医用激光器已形成产业, 商业化产品超过 40 种，年销售额突破 10 亿美元。

与发达国家相比, 我国激光医疗产业在规模、 核心技术、推广应用等方面均存在一定的差距。目 前我国的激光医疗设备以进口为主，关键设备的 国产化比例较小，如国外企业基本垄断了高端眼 科治疗设备。国产激光医疗设备以 $\mathrm{CO}_{2}$ 激光器、 $\mathrm{Nd}: Y A G$ 激光器、半导体激光器为主体, 主要应用 方向包括皮肤外科、通用外科手术、泌尿科、心血 管疾病等, 仍缺乏眼科激光设备、检测与诊断类设 备。在皮肤和泌尿外科方向, 相关国产设备已有所 应用, 但企业规模较小、产品线单一。目前, 我国 激光医疗器械产业布局集中在北京、上海、广东、 湖北、吉林等地区, 注册的激光医疗器械企业约有 200 家。

同时也要注意到, 近来年我国激光技术医疗应 用方面的基础研究和技术创新发展迅速。2019年度 国家自然科学基金国家重大科研仪器研制项目共有 82 项，其中 16 项与激光医疗相关，相应资助金额 为 11857.87 万元，约占全部资助金额的 $20.44 \%$ 。一 批国产医用激光器企业注重技术研发, 不同层次激 光功率、稳定性、准确性等方面的关键性技术取得 突破, 激光医疗器械的国产化进程稳步加速。另外, 国产医用激光器在功率和核心零部件的研发方面也 取得显著进展。随着我国科技创新能力的持续增强 和激光关键技术的整体性突破, 我国医用激光器具 
有广阔的应用前景，相应的产业发展态势如下。

(1) 我国激光医疗产业以中小型民营企业为主, 日益成为创新创业较为活跃的市场领域。

(2) 我国激光医疗产业正由国内销售转向全球 市场, 特别是在 “一带一路” 倡议的带动下, 越来 越多的国内激光医疗器械企业取得了美国食品药品 监督管理局 (FDA)、欧洲统一 (CE) 医疗资质, 出口份额稳步提高。

（3）随着我国注册人制度的实行, 认证和生产、 研发和制造逐步分离, 第三方制造成为发展趋势, 未来将会在激光医疗产业集中地区形成规模化的产 业集群。

（4）随着我国医疗改革推进、居民消费升级、 生活品质提高, 国内医疗市场对于高品质的激光医 疗器械的需求更为强烈。

\section{四、我国激光技术医疗应用面临的问题}

整体来看, 当前我国激光技术医疗应用处于中 低端水平, 真正来自临床需求的牵引力不足, 激光 器技术的 “拿来主义”比较典型; 原创性设计缺乏, 与临床研究的结合不够深入, 难以提出高标准、高 质量的临床需求。虽然国内在部分方面, 如血管靶 向 PDT 与弱光治疗等基础及临床应用研究方面保 持着相对优势, 但是从激光医疗延伸而来的医疗激 光及其产业化发展明显落后于发达国家。

目前在发达国家，约有 $10 \%$ 15\% 的外科手术 使用激光手术器械代替了传统手术刀或其他手术器 械。随着激光技术在医疗应用领域的拓展, 这一比 例必然继续提高。相比之下, 我国激光医疗产业研 究属于跟踪起步并基本同步发展。在技术壁垒较高 的激光设备, 如眼科激光设备中缺乏有竞争力的国 内企业, 这是由于激光医疗器械的研发周期长、技 术壁垒高、失败风险大, 用于临床的激光医疗技术 及设备研发往往不受资本的青睐。在皮肤科、泌尿、 外科等设备技术难度较低的领域, 国产设备已经形 成一定规模, 占据较大份额的中低端市场。在肿瘤 光动力治疗、弱光医疗、口腔等新兴激光医疗领域, 国内外均未出现设备垄断企业。

目前, 我国激光医疗产业整体处于跟踪模仿 的状态, 技术优势和附加值不高, 高端市场份额 偏低。相应的激光技术医疗应用面临着一些亟待
解决的问题。

(1) 激光医学尚无二级学科。虽然激光医学具 有技术性强、综合性高、覆盖领域广、临床应用普 遍等诸多特点, 但由于缺乏明确的学科属性, 使得 我国教育体系中始终未能建立与激光医疗相对应的 二级学科方向, 从而导致专业人才缺乏、学科发展 滞后。

(2) 产业集中度不高。目前国内约有 200 家激 光医疗器械企业, 以中小民营企业为主; 除了数家 新三板企业, 没有主板上市公司; 多数公司仅经营 1 2 种激光医疗产品, 生产集中度低。

（3）产业结构不合理。国产激光医疗产品集中 在价值链的中低端, 而高技术含量、高附加值产品 极少, 产品因同质化和规模小而导致的薄利现象突 出; 众多企业停留在相互仿制阶段, 缺乏原始创新 和自主创新的能力与意愿。

(4) “产学研” 结合未成规模。激光医疗产业 资源未能与强大智力资源、丰富临床资源进行充分 结合, “产学研” 结合中的企业主体明显缺失, 造 成研究和市场脱节, 已有资源优势难以在产业层面 体现出来。

(5) 国内激光医疗设备上市周期过长。我国 医疗器械上市周期平均为 $3 \sim 5$ 年, 而美国、欧盟 等发达国家和地区的医疗器械上市周期一般仅为 $1 \sim 2$ 年。

\section{五、对策建议}

\section{（一）设置 “激光医学” 二级学科}

激光医学的学科交叉性、激光与生物组织作用 的复杂性、激光治疗的专业性等诸多特点, 对激光 医疗从业人员提出了较高的专业素质要求: 既需要 掌握激光诊疗涉及的专业医学知识, 又需要具备激 光医学的专业背景。我国临床医学一级学科没有下 设激光医学二级学科, 使得临床医学本科生教育中 缺少相关专业课程设置。关于研究生招生, 仅有个 别院校具有激光医学专业的招生资格, 且因没有设 立二级学科而只能挂靠其他学科招生。这些因素导 致我国激光医学面临着人才数量短缺、从业人员结 构复杂且学历参差不齐的现状。

激光医学逐渐发展成为了一门独具特色、较为 完整而又朝气蓬勃的前沿交叉学科。随着激光医学 
与临床医学的逐渐融合, 当前的医学院校人才培养 模式已难以匹配国家对激光医疗高级专业人才的鲜 明需求。建议在临床医学一级学科下, 设置 “激光 医学” 二级学科, 构建完整的本科 - 硕士 - 博士培 养体系, 这是促进 “医工” 交叉融合、保障我国激 光医学长远发展的重要措施。

\section{（二）建设激光医学科技创新平台}

以重点和特色临床医院为依托单位, 集中高等 院校、科研院所、高新企业的各自优势, 组建国家 级激光医疗临床转化工程研究中心。中心将区别于 相关光电类的国家级重点实验室, 而以临床实际需 求为牵引, 聚集国内优势研究力量, 注重与企业进 行密切合作以推动产业化发展, 重点攻关激光诊断、 激光治疗和激光监测涉及的核心关键技术, 助力我 国激光诊疗技术的临床转化、示范和推广。

\section{（三）加快推进医疗器械法规建设}

建议相关医疗器械法规将工作机理明确、方案 设计定型、生产工艺成熟、无严重不良事件记录的 激光医疗设备 (如用于眼科和皮肤科的光学相干成 像设备）列入 《免于进行临床试验医疗器械目录》, 进一步降低成熟度高、风险较低产品在临床试验方 面的要求，使企业投入更多精力用于新型产品研发 和质量保障。全面推进医疗器械注册人制度, 实行 产证分离, 为激光医疗器械加速产品创新、缩短研 发周期提供规范便利的环境。加大知识产权保护力 度并落实专利法规, 保护激光医疗器械创新成果以 激发创新意愿。

\section{（四）扶持激光医疗器械相关产业}

激光医疗器械的研发周期长、技术壁垒高、失 败风险大，使得社会资本投资谨慎，因而政府政策 方面的扶植和支持尤为关键。建议地方政府支持建 立激光医疗产业园区，发挥当地的高等院校和科研 院所研究实力、生产制造能力以及产品军民共用的 潜力, 给予必要的资金扶持、税收减免等优惠政策, 促进激光医疗器械产业的集群化、规模化发展。面 向激光医疗产业园区和激光医疗器械第三方制造平 台，给予土地、资金、税收、人才等方面的政策支 持并保持连续性；提供与产业发展相配套的财务和 法律公共服务, 推行公共资源的共享和高效使用。
注重实质性关键技术突破和产业中长期稳健发展, 培育知名品牌和龙头企业。

\section{（五）强化关键核心技术研发攻关}

针对临床亟需, 凝练激光医疗产业 “卡脖子” 问题。我国用于临床的激光医疗技术与国际先进水 平还存在差距, 如国产飞秒激光器的稳定性和安全 性还未达到医疗标准, 国产超快激光器的关键零件 较多依赖进口 (如啁啾镜、非线性晶体等)。建议 发挥市场经济条件下关键核心技术攻关新型举国体 制的优势，通过国家级基金项目的持续支持，突破 激光技术医疗应用的基础性瓶颈，着力解决极具潜 力的激光医疗产业关键核心技术。

\section{参考文献}

[1] Peng Q, Juzeniene A, Chen J Y, et al. Lasers in medicine [J]. Reports on Progress in Physics, 2008, 71(5): 1-28.

[2] Yun S H, Kwok S J. Light in diagnosis, therapy and surgery [J]. Nature Biomedical Engineering, 2017, 1(1): 1-16.

[3] Swanson E A, Fujimoto J G. The ecosystem that powered the translation of OCT from fundamental research to clinical and commercial impact [J]. Biomedical Optics Express, 2017, 8(3): $1638-1664$

[4] Lin L, Hu P, Shi J H, et al. Single-breath-hold photoacoustic computed tomography of the breast [J]. Nature Communications, 2018, 9(1): 1-9.

[5] Boas D A, Dunn A K. Laser speckle contrast imaging in biomedical optics [J]. Journal of Biomedical Optics, 2010, 15(1): $1-12$.

[6] Sun Y, You S, Tu H, et al. Intraoperative visualization of the tumor microenvironment and quantification of extracellular vesicles by label-free nonlinear imaging [J]. Science Advances, 2018, 4(12): $1-10$.

[7] Guitera P, Menzies S W, Longo C, et al. In vivo confocal microscopy for diagnosis of melanoma and basal cell carcinoma using a two-step method: Analysis of 710 consecutive clinically equivocal cases [J]. Journal of Investigative Dermatology, 2012, 132(10): 2386-2394.

[8] Orringer D A, Pandian B, Niknafs Y S, et al. Rapid intraoperative histology of unprocessed surgical specimens via fibre-laser-based stimulated Raman scattering microscopy [J]. Nature Biomedical Engineering, 2017, 1(2): 1-13.

[9] Anderson R R, Parrish J A. Selective photothermolysis: Precise microsurgery by selective absorption of pulsed radiation $[\mathrm{J}]$. Science, 1983, 220(4596): 524-527.

[10] Zaret M M, Breinin G M, Schmidt H, et al. Ocular lesions produced by an optical maser (laser) [J]. Science, 1961, 134(3489): $1525-1526$

[11] Solomon K D, Fernandez de Castro L E, Sandoval H P, et al. LASIK world literature review: Quality of life and patient satisfaction [J]. Ophthalmology, 2009, 116(4): 691-701. 
[12] Allison R R, Moghissi K. Photodynamic therapy (PDT): PDT mechanisms [J]. Clinical Endoscopy, 2013, 46(1): 24-29.

[13] Agostinis P, Berg K, Cengel K A, et al. Photodynamic therapy of cancer: An update [J]. CA: A Cancer Journal for Clinicians, 2011, 61(4): 250-281.

[14] Collins H A, Khurana M, Moriyama E H, et al. Blood-vessel closure using photosensitizers engineered for two-photon excitation [J]. Nature Photonics, 2008, 2(7): 420-424.

[15] Idris N M, Gnanasammandhan M K, Zhang J, et al. In vivo photodynamic therapy using upconversion nanoparticles as remote-controlled nanotransducers [J]. Nature Medicine, 2012,
18(10): 1580-1585.

[16] Chung H, Dai T, Sharma S K, et al. The nuts and bolts of low-level laser (light) therapy [J]. Annals of Biomedical Engineering, 2012, 40(2): 516-533.

[17] Avci P, Gupta A, Sadasivam M, et al. Low-level laser (light) therapy (LLLT) in skin: Stimulating, healing, restoring [J]. Seminars in Cutaneous Medicine and Surgery, 2013, 32(1): 41-52.

[18] Allied Market Research. Medical laser market by product type, application and end user: Global opportunity analysis and industry forecast, 2019-2026 [R]. Portland: Allied Market Research, 2019. 